\title{
Fiducial markers in prostate cancer image-guided radiotherapy
}

\author{
Hamed Ghaffari*1,2, Mahmoud Navaser ${ }^{1}$, Bahram Mofid ${ }^{3}$, Seied Rabi Mahdavi ${ }^{1}$, Reza Mohammadi ${ }^{1}$, \\ Asieh Tavakol ${ }^{4}$
}

\section{Abstract}

Background: Image-guided radiotherapy (IGRT) is recommended to reduce the risk of geometrical miss when modern radiotherapy technologies with high grades of conformity are used. The purpose of this study was to evaluate the efficacy of fiducial markers (FMs) for electronic portal imaging in prostate cancer radiotherapy in term of evaluating the complications associated with FMs implantation, quantifying inter-fraction prostate motion, and determination of optimal planning target volume (PTV) margins.

Methods: In this single institution, prospective, consecutive study, 27 patients underwent implantation of three-gold seed FMs into the prostate gland before prostate radiotherapy. Prior to computed tomography planning, all patients were asked to report any complication associated with FMs implantation that have experienced to date. Daily pre-treatment electronic portal images were captured, and prostate position errors were corrected if they were greater than $2 \mathrm{~mm}$ along three translational directions. Optimal PTV expansions were computed using van Herk formula [PTV-margin $=2.5 \Sigma+0.7 \sigma]$.

Results: FMs implantation was successful with an acceptable toxicity profile in all patients. Without IGRT, margins of $5.4 \mathrm{~mm}, 5.8$ $\mathrm{mm}$ and $5.5 \mathrm{~mm}$, in vertical, longitudinal and lateral directions, respectively, are needed for a $95 \%$ confidence level of complete clinical target volume (CTV) coverage in each treatment session. The PTV margins of $3.0 \mathrm{~mm}, 3.3 \mathrm{~mm}$ and $4.0 \mathrm{~mm}$ in corresponding directions were calculated when FMs based electronic portal imaging was applied.

Conclusion: FMs based electronic portal imaging is an effective tool for prostate cancer IGRT.

Keywords: Fiducial markers, Prostate cancer, Radiotherapy, IGRT

Conflicts of Interest: None declared

Funding: None

*This work has been published under CC BY-NC-SA 1.0 license.

Copyright $₫$ Iran University of Medical Sciences

Cite this article as: Ghaffari H, Navaser M, Mofid B, Mahdavi SR, Mohammadi R, Tavakol A. Fiducial markers in prostate cancer image-guided radiotherapy. Med J Islam Repub Iran. 2019 (11 March);33:15. https://doi.org/10.47176/mjiri.33.15

\section{Introduction}

External beam radiation therapy (EBRT) is a wellestablished method of treating localized prostate cancer patients (1). The goal of radiotherapy (RT) is to deliver maximum dose of the radiation to the target (cancerous tissue), and at the same time, minimizing the radiation dose to the normal tissue surrounding the tumor. New RT techniques such as three-dimensional conformal RT (3DCRT) and intensity modulated RT (IMRT), provide a

Corresponding author: Hamed Ghaffari, hamedghaffari@yahoo.com

1. Department of Medical Physics, School of Medicine, Iran University of Medical Sciences, Tehran, Iran

2. Student Research Committee, School of Medicine, Iran University of Medical Sciences, Tehran, Iran

3. Department of Radiation Oncology, Shohada-e-Tajrish Medical Center, Shahid Beheshti University of Medical Sciences, Tehran, Iran

4. Department of Medical Physics, Roshana Radiotherapy Cancer Center, Tehran, Iran high conformal dose delivery to the target and spare normal tissues $(2,3)$. The first step in RT treatment planning is the acquisition of computed tomography (CT)planning that the CT images have a main role in RT treatment planning. In RT, one of the most important problems is the reproducibility of patient and target position for each RT treatment fraction similar to CT-planning position (4). Any difference between the planned and de-

\section{$\uparrow$ What is "already known" in this topic:}

Reproducibility of the target position in radiotherapy sessions has a central role in the prostate radiotherapy. Electronic portal images can help in evaluating the position of the treatment site, but it does not display soft tissue target.

\section{$\rightarrow$ What this article adds:}

This work strengthens the evidence supporting the efficacy of fiducial markers based image guided prostate radiotherapy. An application of online position verification was an effective protocol to determine optimal planning target volume margin. 
livered position of treatment results in reducing the therapeutic ratio. The planning target volume (PTV) is defined as a margin around the clinical target volume (CTV) to account for patient positioning errors, beam alignment, and organ movement $(5,6)$.

As demonstrated in randomized trials, the use of high doses in prostate EBRT improves biochemical relapsefree survival. However, the incidence of acute and late RT-induced toxicity may increase $(7,8)$. Using a tight CTV-PTV margin is an effective method to reduce these toxicities. A concern with the use of narrow PTV margin is an increased risk of geographical miss because the prostate position can vary owing to day-to-day (inter-fraction motion) relative to the skin tattoos and marks, and also internal prostate motion within a treatment session (intrafraction motion).

Attempt to decrease the risk of geometrical risks, involved image-guided RT (IGRT). A widespread available IGRT system is electronic portal imaging device (EPID) that provides electronic portal images (EPIs) before RT treatment and shows treatment site, and also it allows to correct any discrepancy between the planned and actual treatment position based on bony landmarks (9). EPIs are captured by mega voltage (MV) photons, and in this range of energy, Compton interaction is predominant; therefore, EPIs have a poor soft tissue contrast (4). In order to verify the prostate position using EPIs, it is necessary to have a surrogate of the prostate position. The implantation of radiopaque fiducial markers (FMs) into the prostate can be a proxy of the prostate. The purpose of this study was to evaluate the efficacy of FMs based electronic portal imaging in prostate cancer RT in term of evaluating the complication associated with FMs implantation, quantifying inter-fraction prostate motion, and determination of optimal PTV margins.

\section{Methods}

Twenty-seven patients with localized prostate cancer were included into a single institution, prospective, consecutive study between September 2017 and February 2018. All patients treated with 3DCRT.
Prior to RT, all patients underwent local anesthesia before FMs implantation. Three-gold seed FMs were implanted into the prostate gland under transrectal ultrasound (TRUS) guidance using 17 gauge $20 \mathrm{~cm}$ needle. The gold FMs were implanted in the base, middle and apex of the prostate gland by an expert urologist. After one week, the patients underwent CT-planning. One week is needed to eliminate the infection and inflammation caused by the implantation of the markers. Markers are also fixed during this period. Regarding possible complications after the implantation of fiducial prostate markers, prior to CTplanning, we asked patients to report any complication (fever, hematuria, rectal bleeding, burning sensation and pain), and these were collected using a questionnaire (Supplement 1). The pain was assessed using a visual analogue scale (VAS) from 1 to 10 .

Prior to CT-planning and treatment sessions, we advised all patients to empty rectum and have a comfortable full bladder. The full bladder was achieved by drinking $500 \mathrm{cc}$ water, 30-40 min prior to CT-planning and treatment sessions. The empty rectum and full bladder requirements were consistent and enforced in all patients. All CTplanning scans were performed in the supine position. The scans were obtained in a slice thickness of $3 \mathrm{~mm}$ using a CT-scanner (Siemens SOMATOM Sensation 16-Slice Scanner).

All scans were imported into the Varian Eclipse v.13.6 (Varian Medical System Inc, Palo Alto, CA, USA) treatment planning software (TPS). The prostate gland and seminal vesicles were delineated as the CTV. The PTV was empirically defined by an isotropic margin of $5 \mathrm{~mm}$ around the CTV. All patients treated using either 4 or 7field 3DCRT (Varian Clinac-ix, Varian Medical System Inc, Palo Alto, CA, USA) to a total dose of 76-80 Gy in 2 Gy per fraction.

Daily pre-treatment EPIs were obtained using a flat panel amorphous silicon digital portal imaging device with resolution $1024 \times 768$ pixels. This EPID was mounted iso-centrically on the Linear Accelerator. MV EPIs were acquired with a 6-MV photon at a dose rate 100 monitor unit (MU) per minute, and 1-3 MU were deliv-

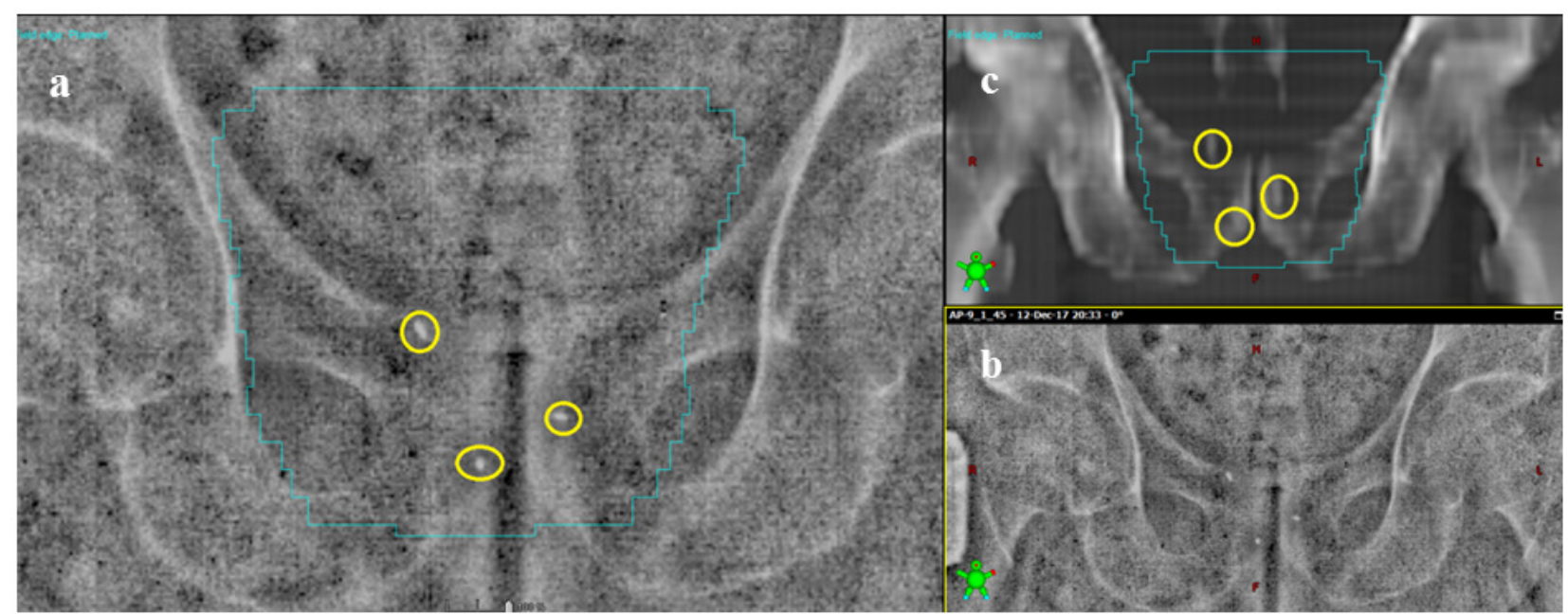

Fig. 1. Electronic portal image of the prostate with implanted three-gold fiducial markers (Figure $2 \mathrm{a}$ and $2 \mathrm{~b}$ ) and corresponding digitally reconstructed radiograph (Figure $2 \mathrm{c}$ ) 
ered per field for portal acquisition. Two orthogonal $\left(0^{\circ}\right.$ and $90^{\circ}$ ) EPIs were obtained in the treatment position prior to treatment, and electronically superposed with the corresponding digitally reconstructed radiographs (DRRs) (as reference images that were created by TPS) using the anatomy matching software (ARIA-record \& verify system). Figure 1 shows FMs based electronic EPIs and corresponding DRR. For studying the inter-fraction prostate motions, the shifts of FMs in two translational directions were evaluated in each field. The online correction of deviation of the FMs was performed by the treatment couch displacement in three translational dimensions before treatment in all patients. The action level $<2 \mathrm{~mm}$ in the all translational direction was empirically used. On the other hand, the displacements less than $2 \mathrm{~mm}$ were not corrected. The fiducial prostate marker deviations were assessed in the three translational directions, vertical (anterior-posterior (AP)), longitudinal (superior-inferior (SI)) and lateral (left-right (LR)).

The formula suggested by van Herk et al. (10) was applied to calculate the fiducial prostate marker deviations for the random ( $\left.\sigma_{\text {population }}\right)$ and the systematic ( $\left.\Sigma_{\text {population }}\right)$ errors, and calculated the CTV-PTV margins in the three translational dimensions.

van Herk et al. defined $\sigma_{\text {population }}$ and $\Sigma_{\text {population }}$ for the deviations of actual position in detail as follows (10, 11):

$X=\frac{1}{N} \sum_{i=1}^{N} x$

$\sigma=\sqrt{\sum_{i=1}^{N} \frac{\left(x_{i}-X\right)^{2}}{N-1}}$

$M=\frac{1}{P} \sum_{i=1}^{P} X_{i}$

$\Sigma_{\text {population }}=\sqrt{\sum_{i=1}^{P} \frac{\left(X_{i}-M\right)^{2}}{P-1}}$

$\sigma_{\text {population }}=\sqrt{\frac{\left(\sigma_{1}{ }^{2}+\cdots+\sigma_{P}{ }^{2}\right)}{P}}$

Where $\mathrm{N}$ is the total number of measured fractions, $\mathrm{x}_{\mathrm{i}}$ is the measured displacement of a patient during each fraction along a specific direction, $\mathrm{X}$ is the mean of the measured displacements of a patient along a specific direction, $\sigma$ is the standard deviation (SD) of the measured displacements of a patient along a specific direction, $X_{i}$ is individual mean setup errors along a specific direction, $M$ is the overall mean of the mean of the measured displacements of all patients in a specific direction, $\Sigma_{\text {population }}$ and $\sigma_{\text {population }}$ are the SD of the systematic and random errors in the prostate position, derived from the FMs, respectively.

At final, the CTV-PTV margins were calculated using van Herk's formula (10):

PTV-margin $=2.5 \Sigma+0.7 \sigma$
This formula assumes that $90 \%$ of patients in the population receive a minimum cumulative $\mathrm{CTV}$ dose of at least $95 \%$ of the prescribed dose.

\section{Results}

The implantation of FMs into the prostate was successful in all cases. Table 1 shows the incidence of the complications after the implantation of FMs into the prostate gland. The mean (range) pain score associated with FMs insertion was 4.33 (2 to 8).

In the current study, data of 27 patients and 694 fractions (approximately 26 fractions per patient) were analyzed. Figure 2 displays the isocenter shift on the pretreatment EPIs relative to the intended position of the isocenter on the corresponding DRRs, along the SI and AP

Table 1. Complications associated with fiducial markers implantation into the prostate gland

\begin{tabular}{lc}
\hline Complication & No. of patient $(\%)$ \\
\hline Fever & $2(7.4)$ \\
Haematuria & $3(11.1)$ \\
Rectal bleeding & $3(11.1)$ \\
Burning sensation & $6(22.2)$ \\
\hline
\end{tabular}

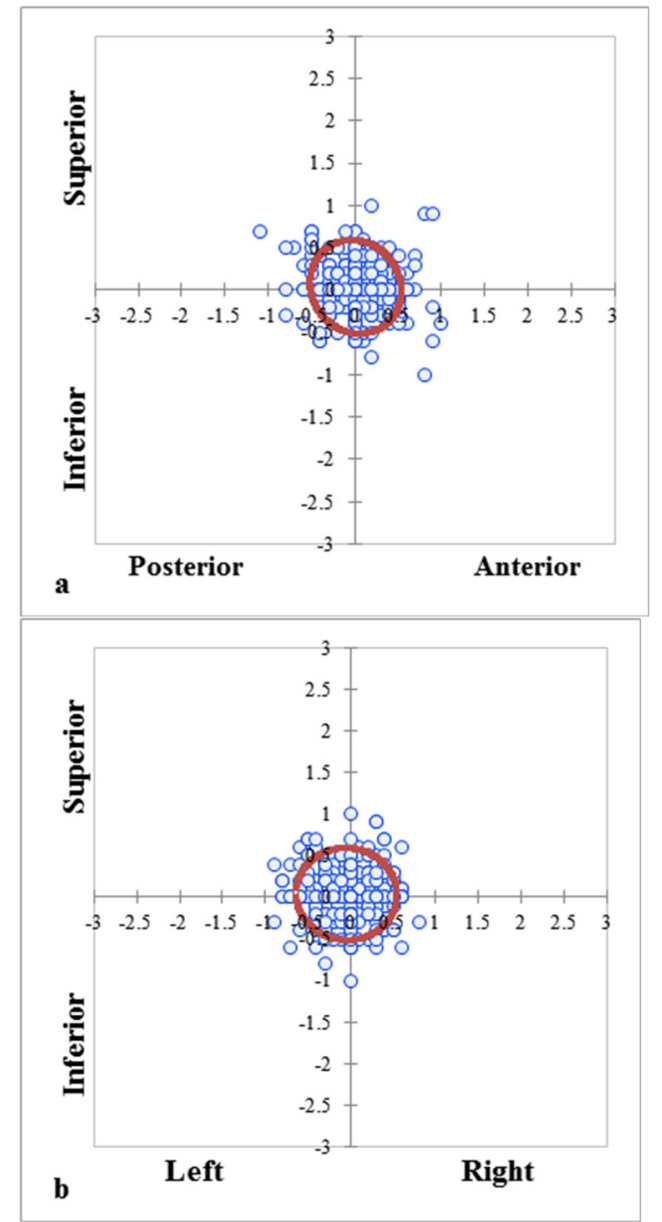

Fig. 2. Isocenter shifts (in $\mathrm{cm}$ ) relative to digitally reconstructed radiograph on pre-treatment electronic portal images $(n=694$ fractions), a) SI and AP directions, and b) SI and LR directions. Ellipse shows $95 \%$ confidence intervals for clinical target volume coverage in each direction. 
(Fig. 2a) and the SI and LR (Fig. 2b) directions. The ellipses in Figures $2 \mathrm{a}$ and $2 \mathrm{~b}$ show $95 \%$ confidence intervals for the CTV coverage in each axis, relative to the DRR. If daily setup verification and correction were not done, the CTV-PTV margins of $5.4 \mathrm{~mm}, 5.8 \mathrm{~mm}$ and $5.5 \mathrm{~mm}$ would be required along the LR, SI and AP directions, respectively, to give a $95 \%$ probability of complete CTV coverage on each given fraction of RT that was given, as shown in Figure $2 \mathrm{a}$ and $2 \mathrm{~b}$.

Figure 3 shows the frequency of the displacements $<2$ $\mathrm{mm}, \leq 5 \mathrm{~mm}$ and $\leq 7 \mathrm{~mm}$. As illustrated in Figure 3, $69.4 \%, 62.7 \%$ and $57.4 \%$ of shifts in the AP, SI and LR directions, respectively, were in the action level. Overall, the CTV-PTV margins of $5 \mathrm{~mm}$ covered approximately $96 \%$ of shifts in all axes.

The overall mean, systematic errors and random errors are outlined in Table 2 . The overall mean values of shifts are $0.22 \mathrm{~mm}, 0.23 \mathrm{~mm}$ and $-0.43 \mathrm{~mm}$ along vertical, longitudinal and lateral directions, respectively. The calculated CTV-PTV margins were $3.0 \mathrm{~mm}, 3.3 \mathrm{~mm}$ and $4.0 \mathrm{~mm}$ in the vertical, longitudinal and lateral axes, respectively.

\section{Discussion}

In our study, image guidance system has been utilized to assess the inter-fraction prostate position errors in 3DCRT delivery. In definitive prostate EBRT, the prostate position verification is important because of the rectum as a critical structure that requires to be spared to decrease the normal tissue complication probability. The results of this study show that the application of implanted fiducial prostate markers and daily pre-treatment EPIs during RT is an effective tool to estimate the extent of the inter-fraction prostate position errors. Although the implantation of FMs into the prostate gland has an invasive procedure, this procedure was well tolerated by the patients with a safe toxicity profile.

As shown in Table 1, FMs implantation was successful with an acceptable toxicity profile in all patients. The patients experienced moderate pain based on VAS of pain after FMs insertion. The mean pain score after implantation of FMs was 4.33. Our data are consistent with pervious published reports (12). We have assessed the complications after one-week implantation of FMs, and did not reassess during RT treatment because the differentiation of the complications associated with FMs insertion and RT was difficult.

From our results, it can be seen that daily FMs based electronic portal imaging and an action level based correction process would have resulted in reducing the PTV margins, from $5.4 \mathrm{~mm}, 5.8 \mathrm{~mm}$ and $5.5 \mathrm{~mm}$ to $3.0 \mathrm{~mm}$, $3.3 \mathrm{~mm}$ and $4.0 \mathrm{~mm}$ in the vertical, longitudinal and lateral directions, respectively. The use of action level for adjusting patient's position if is necessary before the radiation delivery, to correct isocenter shifts of $2 \mathrm{~mm}$ or greater in three translational directions, effectively decreased both systematic and random errors within $1.0 \mathrm{~mm}$ and 3 $\mathrm{mm}$ along three axes, respectively. Daily electronic portal imaging combined to fiducial gold markers can provide an objective method for verifying and correcting the prostate position immediately before RT. In addition, this strategy improves the precision and accuracy of prostate RT.

In a comprehensive review article, it has reported that the systematic and random errors in the routine clinical practice can be less than $2.5 \mathrm{~mm}$ (1SD) for the prostate (13). As shown in Table 2, our data are in good agreement with the study mentioned above (13). Our results allowed us to prove if online verification protocol has not been

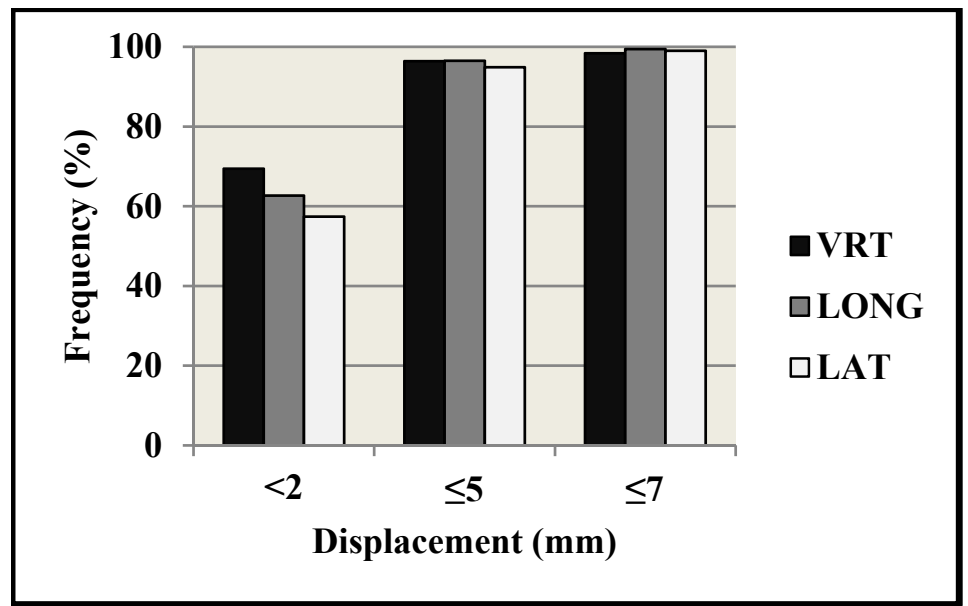

Fig. 3. Frequency of prostate position shifts less than $2 \mathrm{~mm}$ and less than or equal to $5 \mathrm{~mm}$ and $7 \mathrm{~mm}$ for three translational directions (VRT: vertical; LONG: longitudinal; LAT: lateral)

Table 2. Overall mean, systematic error, random error, and PTV margin

\begin{tabular}{lccc}
\hline \multicolumn{4}{c}{ Directions } \\
\hline & VRT $(\mathrm{mm})$ & LONG $(\mathrm{mm})$ & LAT $(\mathrm{mm})$ \\
Overall mean $(\mathrm{M})$ & 0.22 & 0.23 & -0.43 \\
Systematic error $(\Sigma)$ & 0.54 & 0.70 & 0.90 \\
Random error $(\sigma)$ & 2.20 & 2.20 & 2.30 \\
PTV-margin & 3.00 & 3.30 & 4.00 \\
\hline Abbreviations: VRT $=$ vertical; LONG $=$ longitudinal; LAT $=$ lateral; PTV = planning target volume
\end{tabular}


done, nearly $5 \%$ of the daily fractions would have had shifts greater than $5 \mathrm{~mm}$ in any direction. Thus, these geometrical misses can be translated into the reduction in the tumor control rate. Several studies have reported that the CTV-PTV margin of $5 \mathrm{~mm}$ can be an optimum margin in the prostate RT when online position verification combined to FMs based x-rays imaging was applied (14-16). Skarsgard et al. using the implanted gold markers into the prostate and daily electronic portal imaging evaluated the inter-fraction prostate position uncertainties, and also reported the CTV-PTV margins of $3.7 \mathrm{~mm}, 3.7 \mathrm{~mm}$ and 3.6 $\mathrm{mm}$ in the vertical, longitudinal and lateral directions, respectively (14). As outlined in Table 2, our calculated CTV-PTV margins are in line with Skarsgard et al. study (14). However, the current study and reports mentioned above considered the inter-fraction prostate motion, and the calculated PTV margins achieved from fiducial gold marker shifts can only correct the geometric uncertainties. Several factors can lead to the intra-fraction prostate motion, including pulsating arteries and movement of the bowel. A value close to $3 \mathrm{~mm}$ was estimated to account for the intra-fraction prostate movements in IMRT sessions (17). It should be noted that treatment time in each IMRT fraction is approximately $20-30 \mathrm{~min}$, and the intrafraction prostate motion is time-dependent and increases with arising treatment time (18). With regard to treatment modality, we treated all patients with 3DCRT technique using standard dose fractionation regimens (2 Gy per fraction) that treatment time (approximately $10 \mathrm{~min}$ ) is significantly less than IMRT technique. Therefore, we should add at least $2 \mathrm{~mm}$ for accounting intra-fractional motions. In the vertical direction, we have intended to maintain the currently used CTV-PTV expansion of $5 \mathrm{~mm}$. In the longitudinal and lateral axes, we have decided to increase the presently used margin of $5 \mathrm{~mm}$ to $6 \mathrm{~mm}$.

The online correction procedure (taking a pre-treatment EPI, matching it to the corresponding DRR and correcting the patient's position with treatment couch movement) added about $5 \mathrm{~min}$ to the daily treatment setup time. Therefore, the online correction protocol is timeconsuming, and this issue can prevent the widespread application of this protocol at radiation oncology centers with a high workload. Attempt to reduce the cost in term of increased imaging dose to the patient and in-room time, involved offline IGRT correction protocols and weekly $\operatorname{IGRT}(9,19,20)$. With regard to offline protocols, there are two main protocols, including No Action Level (NAL) and Shrinking Action Level (SAL) strategies (9). These protocols can determine the individual systematic error, and thus can be decreased it at an acceptable workload. The NAL approach does not use an action level compared with the SAL approach. The accuracy of the NAL protocol is higher than the SAL protocol. In addition, the NAL protocol is more efficient than the SAL protocol in systematic error reduction. In the NAL protocol, required portal images are less than the SAL protocol that result in reduction of workload and radiation dose to normal tissue. In a radiation oncology department with a high workload, offline protocols may be more efficient strategies because they reduce image acquisition and analysis procedures.
With offline strategy, the random errors are not corrected. Compared with offline correction procedures, online protocol corrects both systematic and random errors (19).

Recently, several researchers have evaluated whether the frequency of taken image for target verification can be decreased from daily to every few days or weekly without a significant reduction in relevant advantage (20-22). In prostate RT, the data revealed that reducing the frequencies of online corrections led to an increase in the PTV volume by approximately $30-40 \%$, and can significantly increase the risk of radiation-induced rectal toxicity (22). A recent phase III multicenter randomized trial showed that the risks of recurrence and late RT-induced rectal injury were significantly reduced by daily online IGRT procedure in prostate cancer. But daily IGRT control increased the risk of second cancers (20). Our data also shows that online correction protocol results in the prostate position verification in high accuracy, but at the cost of a significantly larger effort. A concern with daily x-ray based IGRT systems is an increase in the radiation dose to the patient. A mean dose close to $1.2 \mathrm{cGy}$ per fraction can be delivered to the patient when using portal images. In the current study, we have analyzed near 26 fractions per patient and an average total dose of 31.2 cGy was delivered to the patients by taking EPIs. Overall, online position verification with fiducial prostate marker based electronic portal imaging has remarkable benefits that far outweigh the drawbacks.

A drawback of FMs based IGRT is that all patients cannot receive FMs. Patients with bleeding disorders, prothrombin time (PT)/partial thromboplastin time (PTT) more than 1.5 times the upper limit of normal, platelets less than 50000 and patient with artificial heart valves are contraindication with implantation of FMs (23). There are different types of prostate IGRT systems, including ultrasound, FMs-based x-ray imaging, cone-beam CT (CBCT), Calypso 4D and Cine-magnetic resonance imaging (MRI) $(9,23)$. Some patients are ineligible to receive Calypso 4D and Cine-MRI because these systems are electromagnetic, and patients with pacemakers, implanted ferromagnetic metallic foreign and large metal implants close to the prostate cannot use these IGRT systems. Among different prostate IGRT system, CBCT and ultrasound can be used for everyone (23). Initially, FMs-based x-ray imaging was the gold standard for prostate IGRT. Moseley et al. have demonstrated that CBCT and FMs have comparable localization accuracy in the prostate cancer RT (24).

Authors have considered some potential possible limitations of this study. The rotational prostate position errors were not assessed in this study. Although the incidence of these errors may be low, they exist. Another limitation of this study is not considering the residual errors because a second verification EPI after the repositioning was not done.

\section{Conclusion}

FMs implantation was successful with minimal toxicity and patients were satisfied with this procedure. FMs are a good proxy of the prostate gland position. Online verification protocol combined with FMs based electronic portal 
imaging is an effective IGRT tool that can determine the optimal CTV-PTV expansion, and increase the precision prostate RT, which in turn can decrease radiation dose to the normal tissue. Despite the advent of advanced IGRT systems, such as cine-MRI and Calypso 4D guided RT, these technologies cannot apply to some patients. Therefore, FMs based x-ray imaging has a secure future.

\section{Acknowledgments}

We are thankful to the staff of the Roshana Radiotherapy Cancer Center.

\section{Conflict of Interests}

The authors declare that they have no competing interests.

\section{Research involving human participants and/or animals}

This study involved human participant, and it was conducted considering ethic responsibilities according to the World Medical Association and the Declaration of Helsinki.

\section{Informed consent}

Informed consent was obtained from patients prior to their inclusion in the study.

\section{References}

1. Al-Mamgani A, Heemsbergen WD, Peeters ST, Lebesque JV. Role of intensity-modulated radiotherapy in reducing toxicity in dose escalation for localized prostate cancer. Int J Radiat Oncol Biol Phys. 2009;73:685-91.

2. Zelefsky MJ, Kollmeier M, Cox B, Fidaleo A, Sperling D, Pei X, et al. Improved clinical outcomes with high-dose image guided radiotherapy compared with non-IGRT for the treatment of clinically localized prostate cancer. Int J Radiat Oncol Biol Phys. 2012;84:1259.

3. Mahdavi SR, Ghaffari H, Mofid B, Rostami A, Reiazi R, Janani L. Rectal retractor application during image-guided dose-escalated prostate radiotherapy. Strahlentherapie und Onkologie. 2019 https://doi.org/10.1007/s00066-019-01445-6

4. Stanley S, Griffiths S, Sydes MR, Moore AR, Syndikus I, Dearnaley DP. Accuracy and Reproducibility of Conformal Radiotherapy using Data from a Randomised Controlled Trial of Conformal Radiotherapy in Prostate Cancer (MRC RT01, ISRCTN47772397). Clin Oncol. 2008;20(8):582-90.

5. Jones D. ICRU Report 50-Prescribing, Recording and Reporting Photon Beam Therapy. Med Physic. 1994;21:833-4.

6. Landberg T, Chavaudra J, Dobbs J, Gerard JP, Hanks G, Horiot JC, et al. Report 62. Journal of the International Commission on Radiation Units and Measurements. 1999; os32:NP-NP

7. Zelefsky MJ, Yamada Y, Fuks Z, Zhang Z, Hunt M, Cahlon O, et al. Long-term results of conformal radiotherapy for prostate cancer: impact of dose escalation on biochemical tumor control and distant metastases-free survival outcomes. Int J Radiat Oncol Biol Phys. 2008;71(4):1028-33.

8. Kuban DA, Tucker SL, Dong L, Starkschall G, Huang EH, Cheung $\mathrm{MR}$, et al. Long-term results of the M. D. Anderson randomized doseescalation trial for prostate cancer. Int J Radiat Oncol Biol Phys. 2008;70:67-74.

9. Goyal S, Kataria T. Image guidance in radiation therapy: techniques and applications. Radiology research and practice. 2014;2014:705604.

10. van Herk M, Remeijer P, Rasch C, Lebesque JV. The probability of correct target dosage: dose-population histograms for deriving treatment margins in radiotherapy. Int $\mathrm{J}$ Radiat Oncol Biol Phys. 2000;47:1121-35.

11. van Herk M. Errors and margins in radiotherapy. Seminars in radiation oncology. 2004;14:52-64.

12. Saad A, Goldstein J, Lawrence YR, Weiss I, Saad R, Spieler B, et al.
Transperineal implantation of gold fiducial markers (gold seeds) for prostate image-guided radiation therapy: a feasible technique associated with a low risk of complications. J Med Rad Sci. 2015;62:261-6

13. Hurkmans CW, Remeijer P, Lebesque JV, Mijnheer BJ. Set-up verification using portal imaging; review of current clinical practice. J Eur Soc Ther Radiol Oncol. 2001;58:105-20.

14. Skarsgard D, Cadman P, El-Gayed A, Pearcey R, Tai P, Pervez N, et al. Planning target volume margins for prostate radiotherapy using daily electronic portal imaging and implanted fiducial markers. Rad Oncol (London, England). 2010;5:52.

15. Yartsev S, Bauman G. Target margins in radiotherapy of prostate cancer. Br J Radiol. 2016;89:20160312.

16. Tanyi JA, He T, Summers PA, Mburu RG, Kato CM, Rhodes SM, et al. Assessment of planning target volume margins for intensitymodulated radiotherapy of the prostate gland: role of daily inter- and intrafraction motion. Int J Radiat Oncol Biol Phys. 2010;78:1579-85.

17. Boda-Heggemann J, Köhler FM, Wertz H, Ehmann M, Hermann B, Riesenacker $\mathrm{N}$, et al. Intrafraction motion of the prostate during an IMRT session: a fiducial-based 3D measurement with Cone-beam CT. Rad Oncol (London, England). 2008;3:37-

18. Steiner E, Georg D, Goldner G, Stock M. Prostate and patient intrafraction motion: impact on treatment time-dependent planning margins for patients with endorectal balloon. Int J Radiat Oncol Biol Phys. 2013;86:755-61.

19. Murphy MJ, Balter J, Balter S, BenComo JA, Jr., Das IJ, Jiang SB, et al. The management of imaging dose during image-guided radiotherapy: report of the AAPM Task Group 75. Med Phys. 2007;34:4041-63.

20. Crevoisier Rd, Bayar MA, Pommier P, Muracciole X, Pene F, Dudouet $\mathrm{P}$, et al. Daily versus weekly prostate cancer image-guided radiotherapy: A phase 3, multicenter, randomized trial. J Clin Oncol. 2018;36:4-

21. Rudat V, Hammoud M, Pillay Y, Alaradi AA, Mohamed A, Altuwaijri S. Impact of the frequency of online verifications on the patient set-up accuracy and set-up margins. Rad oncol (London, England). 2011;6:101-.

22. Rudat V, Nour A, Hammoud M, Alaradi A, Mohammed A. Imageguided intensity-modulated radiotherapy of prostate cancer: Analysis of interfractional errors and acute toxicity. Strahlenther Onkol: Organ Deutschen Rontgengesellschaft . 2016;192:109-17.

23. Das S, Liu T, Jani AB, Rossi P, Shelton J, Shi Z, et al. Comparison of image-guided radiotherapy technologies for prostate cancer. Am J Clin Oncol. 2014;37:616-23.

24. Moseley DJ, White EA, Wiltshire KL, Rosewall T, Sharpe MB, Siewerdsen $\mathrm{JH}$, et al. Comparison of localization performance with implanted fiducial markers and cone-beam computed tomography for on-line image-guided radiotherapy of the prostate. Int J Radiat Oncol Biol Phys. 2007;67:942-53. 


\section{Supplement 1}

\section{Questionnaire}

Patient ID: ......

After the implantation of the gold seeds for your prostate cancer radiotherapy, did you experience any of the following within one week after the procedure:

1. Did you experience symptoms of a burning sensation when you passed urine? YES

$\mathrm{NO}$

2. Did you experience any episodes of fevers? YES NO

3. Did you experience symptoms of haemeturia? YES NO

4. Did you experience pain? (If yes, please scale it based on visual analogue scale (VAS) from 1 to 10 as to be seen below). YES

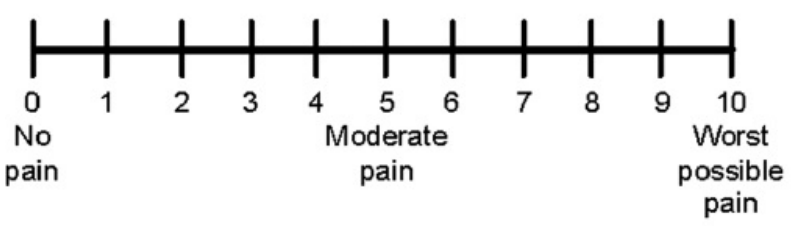

5. Did you experience rectal bleeding when you passed stool? YES

NO 